\title{
The Hydrodynamic Environment for the s Process in the He-Shell Flash of AGB Stars
}

\author{
Paul R. Woodward ${ }^{* a}$, David H. Porter ${ }^{a}$, Falk Herwig ${ }^{b, c}$, Marco Pignatari ${ }^{c}$, Jagan \\ Jayaraj $^{a}$, and Pei-Hung Lin ${ }^{a}$ \\ ${ }^{a}$ University of Minnesota, Laboratory for Computational Science \& Engineering 499 Walter \\ Library, 117 Pleasant St. S. E., Minneapolis, Minnesota 55455, USA \\ ${ }^{b}$ University of Victoria, Dept. of Physics and Astronomy, Victoria, BC, V8W $3 P 6$ Canada \\ ${ }^{c}$ Keele University, Astrophysics Group, North Staffordshire, ST5 5BG, UK \\ E-mail: paul@lcse.umn.edu, fherwig@uvic.ca
}

The He-shell flash convection in AGB stars is the site for the high-temperature component of the s-process in low- and intermediate mass giants, driven by the ${ }^{22} \mathrm{Ne}$ neutron source. During this phase several s-process branchings are activated, including some with time scales similar to the convective turn-over time scale. In addition, uncertainties regarding convective mixing at both the bottom and the top of the convective shell are preventing accurate predictions of sprocess yields. The upper convection boundary plays a critical role during the $\mathrm{H}$-ingestion episode that may lead to neutron-bursts in the most metal-poor AGB stars. We address these problems through global 3-dimensional hydrodynamic simulations including the entire spherical He-shell flash convection zone (as oposed to the 3D box-in-a-star simulations). An important aspect of our current effort is to establish the feasibility of our appoach. We explain why we favour the explicit treatment over the anelastic approximation for this problem. The simulations presented in this paper use a Cartesian grid of $512^{3}$ cells and have been run on four 8-core workstations for four days to simulate $\sim 5000$ s, which corresponds to almost ten convective turn-over times. The convection layer extends radially at the simulated point in the flash evolution over $7 \mathrm{H}_{\mathrm{p}}$ pressure scale-heights and exceeds the size of the underlying core. Convection is dominated by large convective cells that fill more than an entire octant. In order to better understand the conditions of the s-process branchings in this environment we have extracted particle tracers, and we discuss the thermodynamic trajectories along those paths.

10th Symposium on Nuclei in the Cosmos

July 27 - August 12008

Mackinac Island, Michigan, USA

\footnotetext{
* Speaker.
} 


\section{Introduction}

The main component of the s process nucleosynthesis originates in AGB stars, and is intimately linked to the mixing induced by convection. In the He-shell flash convection zone of stars with core-masses $>0.6 \mathrm{M}_{\odot}$ the ${ }^{22} \mathrm{Ne}(\alpha, \mathrm{n})^{25} \mathrm{Mg}$ reaction releases a burst of neutrons $\left(N_{\mathrm{n}} \sim\right.$ $\left.10^{11} \mathrm{~cm}^{-3}\right)$. This activates s-process branchings sensitive to the peak temperature reached during the flash, for example at ${ }^{95} \mathrm{Zr}$ [6] or ${ }^{128} \mathrm{I}$ [9], which in turn depen on the hydrodynamic properties of this convection, including its boundaries [4]. Observables derived from such branchings make He-shell flash convection a unique laboratory for investigating hydrodynamic flows in the deep stellar interior where the elements are made.

However, our premier motivation are those He-shell flashes that ingest $\mathrm{H}$ from the overlying, unprocessed and stable layers. These have been found in stellar evolution calculations of AGB stars of extremely low metal content (e.g. [1]), and in other situations [3]. The convective-reactive nature of the $\mathrm{H}$-ingestion flash as well as its dependence on the exact nature of convective boundary mixing, invalidated the one-dimensional stellar evolution approach, as it for example falsly assumes that the abundance of fuel is constant on spheres. This assumption may lead to predictions like a split of convection zones which are most likely artifacts of the inappropriate assumption of spherical symmetry [11]. Here we focus on the entrainment process in full 3D geomertry that leads to the convective-reactive case, thereby improving on previous non-reactive simulations of He-shell flash convection, which where in plane-parallel geometry and only in two dimensions [5]. First we explain why the explicit hydrodynamic treatment is justified for this problem.

\section{Hydrodynamic simulations, computational method and implications for the $s$ process}

The PPM gas dynamics scheme $[10,2,12,13]$ has been adapted over a decade ago to simulate on 3-D Cartesian meshes AGB star envelope convection flows in their spherical geometry [8, 15]. In that work the roughly sonic to supersonic motions near the surface of the star made the explicit formulation of this numerical scheme highly appropriate. In the present work, we adopt a similar computational approach to the simulation of the helium shell flash convection zone in AGB stars.

The principal difficulty introduced through our numerical approach is our code's explicit formulation, which performs each grid cell update using only local data. We follow sound waves in the flow, and these make the flow tend toward hydrostatic equilibrium in the radial dimension without our needing to enforce this constraint explicitly. This feature of our code is a disadvantage only because of its computational cost. Mach numbers in the helium shell flash convection flow tend to range up to about $1 / 30^{1}$, which means that our explicit treatment of sound wave propagation forces us to take many more time steps than would be needed in an anelastic treatment.

However, this numerical approach is affordable because of two aspects. The first is directional operator splitting, that an anelastic simulation on the same uniform Cartesian grid could not employ, because of its much larger time step. This already reduces the the work difference between the two schemes down by a factor of two to three. The reason for this is that directional splitting in

\footnotetext{
${ }^{1}$ This is for convective gusts actually observed in our simnulations, and not for the much smaller mean convective velocity that mixing-length would indicate.
} 

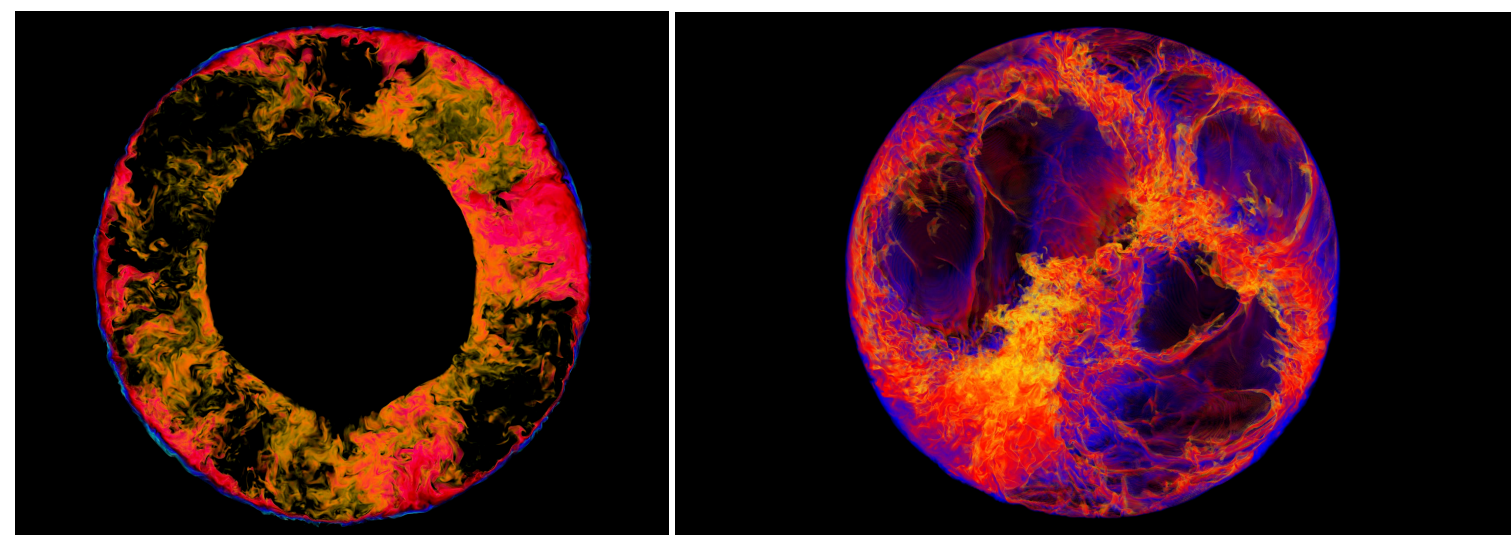

Figure 1: Snapshots of a hemisphere of the AGB star's interior taken from PPM simulations on uniform Cartesian grids of $512^{3}$ cells, corresponding to time $-0.07 \mathrm{yr}$ on the left and $0.2 \mathrm{yr}$ on the right (cf. Fig. 2 in [5]). The outer radius of each image is the upper boundary of the convection zone, located on the left at $15,200 \mathrm{~km}$ and on the right at $30,000 \mathrm{~km}$. The bottom of the convection zone is at $9,150 \mathrm{~km}$ and $10,000 \mathrm{~km}$ in the left and right panel, respectively. As before $[5,11]$ the convection layer is sandwiched by a stable layer inside and outside to capture the behavior of stable oscillatory motions there that are driven by the convection, and artificially damped beyond a certain radius in our simulations. Although we do not expect the H-ingestion to occur in the high-metallicity AGB star that we used as a template for these simulations, we nevertheless chose in both cases the entrained material from above the convection zone to visualise the flows. Concentrations of entrained fluid from above that are near but not quite equal to unity are blue, while red and yellow correspond to concentrations of around $1 \%$ and 0.1 to $0.01 \%$. Both flows display the dominant role of very large convection cells, which tends to invalidate the assumptions of the mixing length theory and to support the need for detailed treatment of these flows in 3-D in order to determine the s-process branching nucleosynthesis. On the left, visualisation focuses on a vertical slice, while on the right the hemisphere facing out of the page has been cut away so that we look from the inside out at the far hemisphere of the star. These images are taken from movie animations that can be viewed from the 'MOVIES' link on the main LCSE web site at http://www.lcse.umn.edu.

an explicit method accurately captures the corner transport for both sound waves and fluid advection with no additional computational labor over a 1-D scheme. The second issue is the implicit Poisson solve, or equivalent, required by the anelastic scheme. On today's large machines, global synchronization - of which many are required per Poisson solve - is the most expensive fundamental operation that can be performed. The second most expensive thing on a modern machine is communication not overlapped with computation, including - and not sufficiently appreciated in the computing community - a CPU's communication with its locally attached main memory. The standard iteration of a Poisson solver offers little computation to perform while the necessary data is being provided to the CPU by the hardware. As a result a Poisson solver will run at a fraction of the Gflop/s/CPU of an explicit gas dynamics scheme like PPM. These factors together practically eliminate the cost advantage of the anelastic scheme at Mach numbers seen in helium shell flash convection flows. In addition, comparisons have shown that perturbations and instabilities at the boundaries are less prounounced and probably underestimated in anelastic simulations compared to the explicit treatment [7]. Since the convective boundaries are so important in our simulations we conclude that the explicit treatment is the superior method for our problem. 

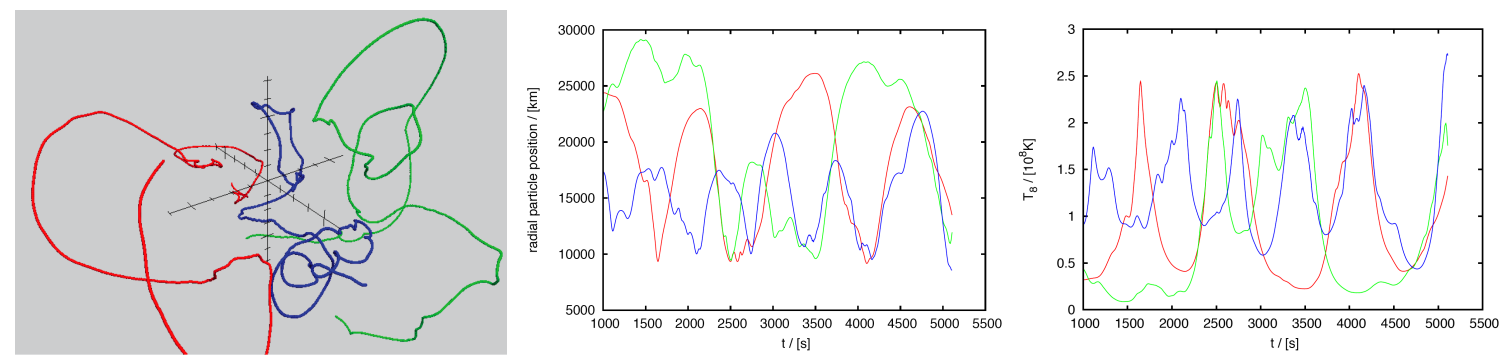

Figure 2: Left: three arbitrary particle traces over 4176s of simulated time in the flow; middle and right: the radial position and the temperature of the same particle traces.

We have implemented our PPM codes on both 4-core Intel CPUs and 8-core IBM Cell processors (cf. [14]). The simulation shown in the right panel of Fig. 1 ran over 80,000 time steps to simulate 5147 seconds of problem time, running for about 4 days on $32 \mathrm{CPU}$ cores in 4 workstations at a sustained performance of $3.4 \mathrm{Gflop} / \mathrm{s} / \mathrm{core}$. An early test of essentially this same two-fluid PPM code ran at $2.64 \mathrm{Gflop} / \mathrm{s} /$ core on $5720 \mathrm{CPU}$ cores (IBM Cell processor cores) in the Roadrunner machine at Los Alamos, delivering 15 Tflop/s, which should improve as we perfect our implementation for the Roadrunner platform. We expect this code to scale linearly to the full Roadrunner machine which has over 100,000 CPU cores. Even if we used only $4 \%$ of that machine we could do this same simulation on a $1024^{3}$ grid and with the correct heat injection rate in just 21 hours, which shows that these computations are eminently affordable.

In the new global simulations of He-shell flash convection, in particular at the later time (right panel in Fig. 1) when the geometric extent of the unstable layer is close to the maximum, the large-scale behaviour of the convection is revealed. Material from above the convection zone is entrained in a large-scale and irregular way. Large cells, that occupy a full quadrant of the thick shell, dominate the flow, indicating that the mixing patterns relevant for T-sensitive branchings may be more complicated.

We can interpolate representative particle paths and the fluid states along those paths. Using the fluid states along these particle paths we can solve a complete nuclear reaction network to obtain the behavior along this path. In the simulation, turbulent mixing of tracked constituents, in this case the original gas of the convection zone and that entrained from the stably stratified region above it, is carefully accounted for using a moment-conserving advection scheme that we call PPB $[12,11]$. The PPB scheme has roughly double or triple the linear resolving power of the PPM advection scheme, which accounts for the level of fine detail that is evident in the results shown in Fig. 1. However, the nuclear reaction network will track a great number of additional concentrations. By following many different particle paths simultaneously, we can use this information to estimate gradients of these concentrations in a manner similar to the interpolation methods of smoothed particle hydrodynamics algorithms. Putting this together with the PPM simulation's detailed information on the turbulent kinetic energy along the particle path, an accurate estimate of s-process yields in this flow can be computed.

\section{Acknowledgments}

This work has been supported by the U. S. Department of Energy through a contract with the 
Los Alamos National Laboratory and a grant from the MICS program of the Office of Science, DE-FG02-03ER25569. It has also been supported by NSF Computer Research Infrastructure grant CNS-0708822, a donation of equipment from IBM, and support from the Minnesota Supercomputing Institute. FH was supported in part by Marie Curie grant MIRG-CT-2006-046520, and JINA.

\section{References}

[1] S. Cassisi, V. Castellani, and A. Tornambe, The Evolutionary Properties and Peculiar Thermal Pulses of Metal-deficient Low-Mass Stars, ApJ 459 (1996), 298.

[2] P. Colella and P. R. Woodward, The Piecewise Parabolic Method (PPM) for Gas-Dynamical Simulations, Journal of Computational Physics 54 (1984), 174-201.

[3] F. Herwig, The evolutionary time scale of Sakurais object: A test for convection theory, ApJ Lett. 554 (2001), L71-L74.

[4] , The evolution of AGB stars, ARAA 43 (2005), 435.

[5] Falk Herwig, Bernd Freytag, Robert M. Hueckstaedt, and Francis X. Timmes, Hydrodynamic simulations of He-shell flash convection, ApJ 642 (2006), 1057-1074.

[6] M. Lugaro, F. Herwig, J. C. Lattanzio, R. Gallino, and O. Straniero, s-Process Nucleosynthesis in Asymptotic Giant Branch Stars: A Test for Stellar Evolution, ApJ 586 (2003), 1305-1319.

[7] C. A. Meakin and D. Arnett, Anelastic and Compressible Simulations of Stellar Oxygen Burning, ApJ 665 (2007), 690-697.

[8] D. H. Porter and P. R. Woodward, Three-dimensional Simulations of Turbulent Compressible Convection, ApJS 127 (2000), 159-187.

[9] R. Reifarth, F. Käppeler, F. Voss, K. Wisshak, R. Gallino, M. Pignatari, and O. Straniero, ${ }^{128} \mathrm{Xe}$ and ${ }^{130}$ Xe: Testing He-Shell Burning in Asymptotic Giant Branch Stars, ApJ 614 (2004), 363-370.

[10] P. Woodward and P. Colella, The numerical simulation of two-dimensional fluid flow with strong shocks, Journal of Computational Physics 54 (1984), 115-173.

[11] P. Woodward, F. Herwig, D. Porter, T. Fuchs, A. Nowatzki, and M. Pignatari, Nuclear Burning and Mixing in the First Stars: Entrainment at a Convective Boundary Using the PPB Advection Scheme, First Stars III, American Institute of Physics Conference Series, vol. 990, March 2008, pp. 300-308.

[12] P. R. Woodward, Numerical Methods for Astrophysicists, Astrophysical Radiation Hydrodynamics (K.-H. Winkler and M. L. Norman, eds.), Reidel, 1986, online at http://www.lcse.umn.edu/projects/34/34_PPMlogoPaper.pdf, pp. 245-326.

[13] _ The PPM Compressible Gas Dynamics Scheme, Implicit Large Eddy Simulation, Computing Turbulent Fluid Dynamics (L. Margolin F. Grinstein and W. Rider, eds.), Cambridge University Press, 2006, Available at http://www.lcse.umn.edu/ILES/PPM-for-ILES-turb-appls-2-26-05-cup6a-10.pdf.

[14] P. R. Woodward, J. Jayaraj, P.-H. Lin, and P.-C. Yew, Moving Scientific Codes to the IBM Cell Processor and Other Multicore Microprocessor CPUs, Computing in Science \& Engineering, 2008, in press. online at http://www.lcse.umn.edu/ILES/PPM-for-ILES-turb-appls-2-26-05-cup6a-10.pdf.

[15] P. R. Woodward, D. H. Porter, and M. Jacobs, 3-D Simulations of Turbulent Compressible Stellar Convection, 3D Stellar Evolution (S. Turcotte, S. C. Keller, and R. M. Cavallo, eds.), Astronomical Society of the Pacific Conference Series, vol. 293, 2003, online at http://www.lcse.umn.edu/3Dstars, pp. $45-+$. 